APS

physics

This is the accepted manuscript made available via CHORUS. The article has been published as:

\title{
Vibrational states of atomic hydrogen in bulk and nanocrystalline palladium studied by neutron spectroscopy
}

Maiko Kofu, Naoki Hashimoto, Hiroshi Akiba, Hirokazu Kobayashi, Hiroshi Kitagawa,

Kazuki lida, Mitsutaka Nakamura, and Osamu Yamamuro

Phys. Rev. B 96, 054304 - Published 24 August 2017

DOI: 10.1103/PhysRevB.96.054304 


\title{
Vibrational states of atomic hydrogen in bulk and nanocrystalline palladium studied by neutron spectroscopy
}

\author{
Maiko Kofu, ${ }^{1, *}$ Naoki Hashimoto, ${ }^{1}$ Hiroshi Akiba, ${ }^{1}$ Hirokazu Kobayashi, ${ }^{2}$ \\ Hiroshi Kitagawa, ${ }^{2}$ Kazuki Iida, ${ }^{3}$ Mitsutaka Nakamura, ${ }^{4}$ and Osamu Yamamuro ${ }^{1, \dagger}$ \\ ${ }^{1}$ Institute for Solid State Physics, University of Tokyo, Kashiwa, Chiba 27r-8581, Japan \\ ${ }^{2}$ Graduate School of Science, Kyoto University, Sakyo-ku, Kyoto 606-8502, Japan \\ ${ }^{3}$ Research Center for Neutron Science and Technology, \\ Comprehensive Research Organization for Science \\ and Society, Tokai, Ibaraki 319-1106, Japan \\ ${ }^{4}$ Materials and Life Science Division, \\ J-PARC Center, Tokai, Ibaraki 319-1195, Japan
}

(Dated: July 12, 2017)

\begin{abstract}
The vibrational states of hydrogen atoms in bulk and nanocrystalline palladium were examined in a wide energy region $0 \leq \hbar \omega \leq 300 \mathrm{meV}$ using neutron spectroscopy. In bulk $\mathrm{PdH}_{0.73}$, the vibrational excitations of $\mathrm{H}$ atoms were roughly reproduced by the quantum harmonic oscillator (QHO) model. In $\mathrm{PdH}_{0.42}$ nanocrystals with a diameter of $8 \mathrm{~nm}$, however, additional vibrational excitations were found at energies above $80 \mathrm{meV}$. The energies and intensities of the additional states were not explained by QHO but reasonably described as vibrations in a highly anharmonic trumpet-like potential. The additional excitations are attributed to the vibrations of $\mathrm{H}$ atoms at tetrahedral sites in the subsurface region stabilized by surface effects. The present work has provided the experimental observation of hydrogen vibration inside metal nanoparticles.
\end{abstract}

\footnotetext{
* Present address: Materials and Life Science Division, J-PARC Center, Tokai, Ibaraki 319-1195, Japan; maiko.kofu@j-parc.jp

$\dagger$ yamamuro@issp.u-tokyo.ac.jp
} 


\section{INTRODUCTION}

The behavior of hydrogen in metals has attracted much attention in fundamental and applied research areas. Palladium hydride $\left(\mathrm{PdH}_{x}\right)$ is a typical metal-hydrogen system and has been intensively studied for many decades. Pd has remarkable abilities to absorb plenty of hydrogen and to catalyze a broad range of chemical reactions. Metal nanoparticles are of current interest owing to their unique properties as compared with bulk materials [1-4]. It has been demonstrated that the $\mathrm{H}$ absorption property of $\mathrm{Pd}$ nanoparticles are markedly different from that of bulk Pd and strongly depends on their size, shape, and surface structure [5-12]. The Pd nanoparticles with high surface area also offer high catalytic activity [13-16]. In the field of surface science, it has been often discussed that the potential energy for the $\mathrm{H}$ sites significantly changes near (a few layers below) the surface of particle, which is usually called "subsurface" [3, 17-24]. A key issue in metal hydride nanoparticles is to clarify the thermodynamic state and structure of subsurface which are closely connected with the $\mathrm{H}$ absorption ability and the high catalytic performance.

Bulk $\mathrm{PdH}_{x}$ undergoes a phase transition from a hydrogen dilute $\alpha$ phase to a concentrated, lattice expanded $\beta$ phase. In both phases, the $\mathrm{H}$ atoms occupy the interstitial octahedral $(O)$ sites $(1 / 2,1 / 2,1 / 2)$ in the face-centered cubic (fcc) Pd lattice [27-37] (see Fig. 1). Our recent neutron diffraction $(\mathrm{ND})$ work on nanocrystalline $\mathrm{PdD}_{x}$ (nano- $\mathrm{PdD}_{x}$ ) demonstrated that some of $\mathrm{H}$ atoms, probably in the subsurface, are accommodated at the tetrahedral $(T)$ sites $(1 / 4,1 / 4,1 / 4)[38]$. It was also reported by a quasielastic neutron scattering (QENS) study that an additional fast diffusion process of the $\mathrm{H}$ atoms appeared in nano- $\mathrm{PdH}_{x}[39]$. These results suggest that the $\mathrm{H}$ atoms diffuse faster near the surface, probably via the $T$ sites.

Inelastic neutron scattering (INS) spectroscopy is a powerful technique in probing vibrational states of $\mathrm{H}$ atoms in metal lattices. The precise analysis of INS spectra enables us to predict the potential surface around the $\mathrm{H}$ atom position. With this view, a number of INS measurements have been performed for bulk $\operatorname{PdA}_{x}(\mathrm{~A}=\mathrm{H}, \mathrm{D}, \mathrm{T})$ so far [40-50]. They have demonstrated that the optical $\mathrm{H}$ vibrations in $\mathrm{PdH}_{x}$ is rather anisotropic and anharmonic [49, 50], which is consistent with theoretical prediction of ab initio calculations [51]. Stuhr et al. have made INS measurements up to $140 \mathrm{meV}$ for nano-PdH $\mathrm{Pd}_{x}(x \leq 0.048)$ with a diameter of smaller than $23 \mathrm{~nm}$ [53]. The vibrational excitations, which are attributed to 


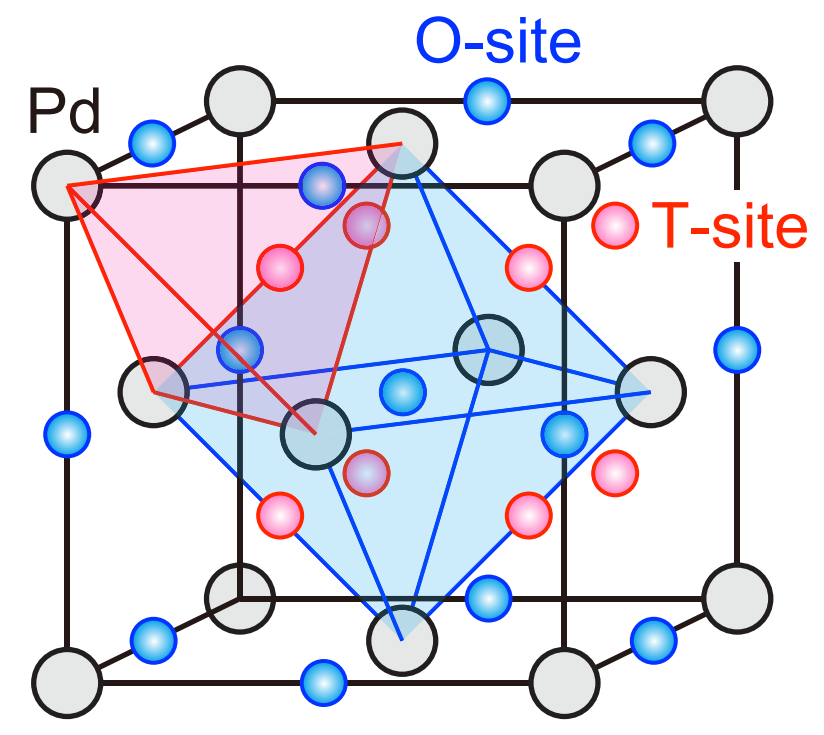

FIG. 1. (Color online) Locations of interstitial hydrogen atoms in an fcc Pd lattice; octahedral (O) and tetrahedral $(\mathrm{T})$ sites.

the $\mathrm{H}$ atoms in the grain boundary and/or the inner surface, were observed in the energy region between $90 \mathrm{meV}$ and $140 \mathrm{meV}$. They also found an excitation peak at 50-80 meV which resembles that observed in bulk $\mathrm{PdH}_{x}$.

Here we report an INS study of bulk $\mathrm{PdH}_{0.73}$ and high-quality nano- $\mathrm{PdH}_{0.42}$ with a smaller and more uniform diameter $(8.0 \pm 0.9 \mathrm{~nm})$ than in the previous study [53]. The vibrational dynamics of $\mathrm{H}$ atoms were investigated in the wide energy-transfer region $0 \leq \hbar \omega \leq 300 \mathrm{meV}$ using a modern sophisticated neutron spectrometer, to gain insight into the shape of the potential well for the hydrogen vibration in the subsurface. Our nanocrystals are capped by a protective polymer, polyvinylpyrrolidone (PVP) and are isolated from each other, in contrast to the Stuhr's nanoparticles. We do not suffer from H atoms in grain boundaries. Thus, this is the first experimental work on hydrogen vibration inside metal nanoparticles.

\section{EXPERIMENTAL}

The Pd powder used as a bulk sample was obtained from Sigma Aldrich with a specified purity of $99.9 \%$ and the hydrogen gas (purity 99.99 \%) from Suzuki Shokan. The $\mathrm{Pd}$ nanocrystals used in this work are basically the same as those in the previous heat capacity [54], neutron diffraction [38], and QENS measurements [39]. The nanocrystals were 
synthesized by the same method descried elsewhere [55]. The mass ratio of Pd and PVP was determined to be 68 : 32, corresponding to the $\mathrm{Pd}$ volume fraction of 0.175 . The sample amounts of the Pd powder and the nanocrystals (including PVP) were ca. $6 \mathrm{~g}$ and $2 \mathrm{~g}$, respectively. The pretreatment and hydrogenation of the samples and the determination of $\mathrm{H}$ concentrations were carried out as described in Ref. 39. The dehydrogenated Pd nanocrystal which were measured to estimate the effect of PVP were prepared by evacuating the nano-PdH sample at $100^{\circ} \mathrm{C}$. The samples were loaded into annular aluminum cells with a thickness of $0.5-1 \mathrm{~mm}$ and a diameter of $18 \mathrm{~mm}$.

Neutron spectra of bulk $\mathrm{PdH}_{0.73}$, nano-Pd, and nano- $\mathrm{PdH}_{0.42}$ were recorded at $10 \mathrm{~K}$ using a time-of-flight Fermi chopper spectrometer, 4SEASONS [56], at the Japan Proton Accelerator Research Complex (J-PARC). The spectrometer has a unique ability to record

the scattering events for neutrons with multiple incident energies $E_{\mathrm{i}}$ simultaneously [57]. The frequency of a Fermi chopper was set to $600 \mathrm{~Hz}$ and the phase was configured to select an $E_{\mathrm{i}}$ set of 942,331 , and $168 \mathrm{meV}$. Here we do not show the data obtained with $E_{\mathrm{i}}=942 \mathrm{meV}$ because the phonon excitations were not separated well due to the coarse energy resolution. The empty cell was measured to subtract scattering contribution from the instrument and the cell. The temperature of the samples was controlled by using a top-loading closed cycle refrigerator. The data reduction was done with Utsusemi software package [58] developed in J-PARC.

\section{RESULTS AND DISCUSSION}

\section{A. Dynamical structure factors}

Figure 2 shows color contour maps of dynamical structure factor, $S(Q, \omega)$, for bulk $\mathrm{PdH}_{0.73}$ and nano- $\mathrm{PdH}_{0.42}$, obtained with $E_{\mathrm{i}}=331 \mathrm{meV}$ at $10 \mathrm{~K}$. Here the contribution from the empty cell and an instrumental background were already subtracted. As for nano$\mathrm{PdH}_{0.42}$, the nano-Pd data were also subtracted. The amount of $\mathrm{H}$ atoms in PVP is nine times larger than that in nano- $\mathrm{PdH}_{0.42}$. The subtraction of scattering from PVP is necessary to extract vibrational excitations of $\mathrm{H}$ atoms in $\mathrm{Pd}$. Figure 3 presents the actual process of the subtraction for the data integrated over limited $Q$ regions. In fact, the observed $S(Q, \omega)$ of nano- $\mathrm{PdH}_{0.42}$ and nano-Pd include large scattering contribution from $\mathrm{PVP}$ as seen in 

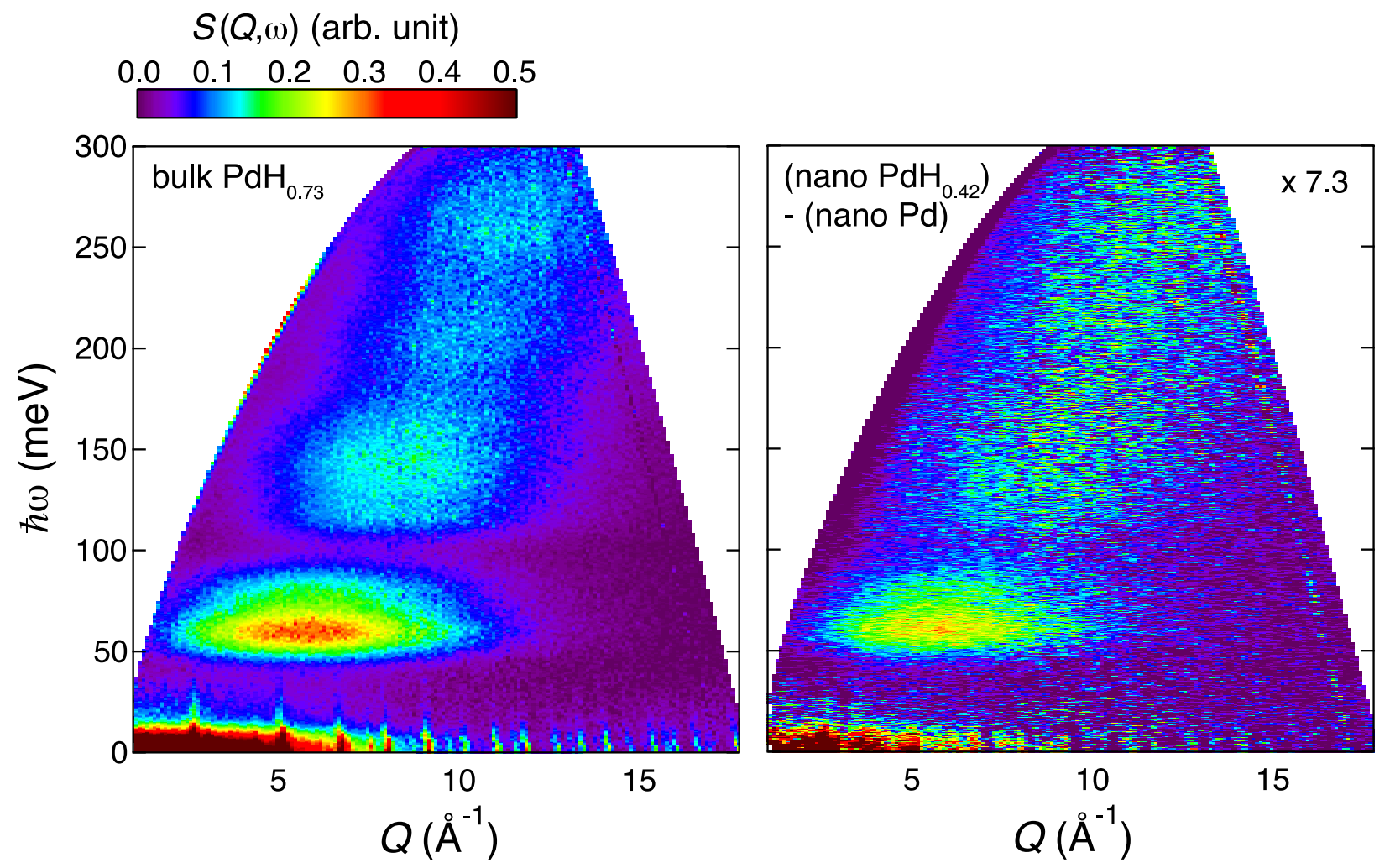

FIG. 2. (Color online) Dynamical structure factors as functions of energy and momentum transfer for bulk and nano- $\mathrm{PdH}_{x}$ recorded at $10 \mathrm{~K}$. The scattering contribution from nano-Pd has been subtracted for nano- $\mathrm{PdH}_{0.42}$. The data were obtained with $E_{\mathrm{i}}=331 \mathrm{meV}$.

Fig. 3. The several peaks between 100 and $140 \mathrm{meV}$ are assigned to the vibration of the C-C bonds in pyrrolidone ring and the strong broad band at around $170 \mathrm{meV}$ to the carbonyl $(\mathrm{C}=\mathrm{O})$ stretch [59]. It is emphasized that the peaks disappear in the subtracted data (filled circles in Fig. 3), indicating successful subtraction.

Vibrational excitations are clearly visible at around 70, 140, 210, and $280 \mathrm{meV}$ in bulk $\mathrm{PdH}_{0.73}$. On the other hand, the $S(Q, \omega)$ map of nano- $\mathrm{PdH}_{0.42}$ exhibits a clear excitation at $70 \mathrm{meV}$ and a continuous excitation above $120 \mathrm{meV}$. The vibration of the host $\mathrm{Pd}$ lattice mainly reflects acoustic phonons which appear below $26 \mathrm{meV}[42,49]$. The excitations observed above $50 \mathrm{meV}$ correspond to multiphonon processes that originate in the vibrations of $\mathrm{H}$ atoms.

Figure 4 displays the comparisons in INS spectra between bulk $\mathrm{PdH}_{0.73}$ and nano- $\mathrm{PdH}_{0.42}$. The spectrum of nano- $\mathrm{PdH}_{0.42}$ exhibits the first excitation peak at around $60 \mathrm{meV}$ as in the case of bulk $\mathrm{PdH}_{0.73}$. A significant difference on the spectra is seen in the higher energy 
(a)

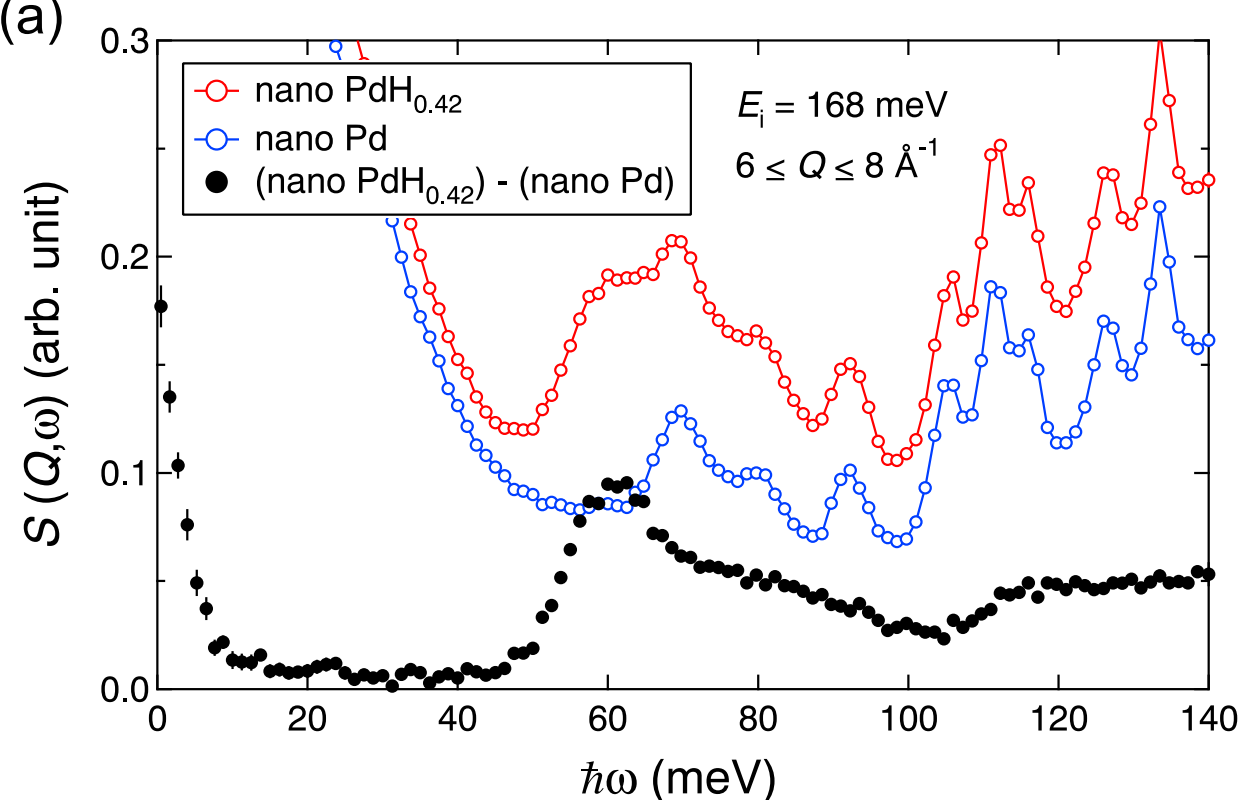

(b)

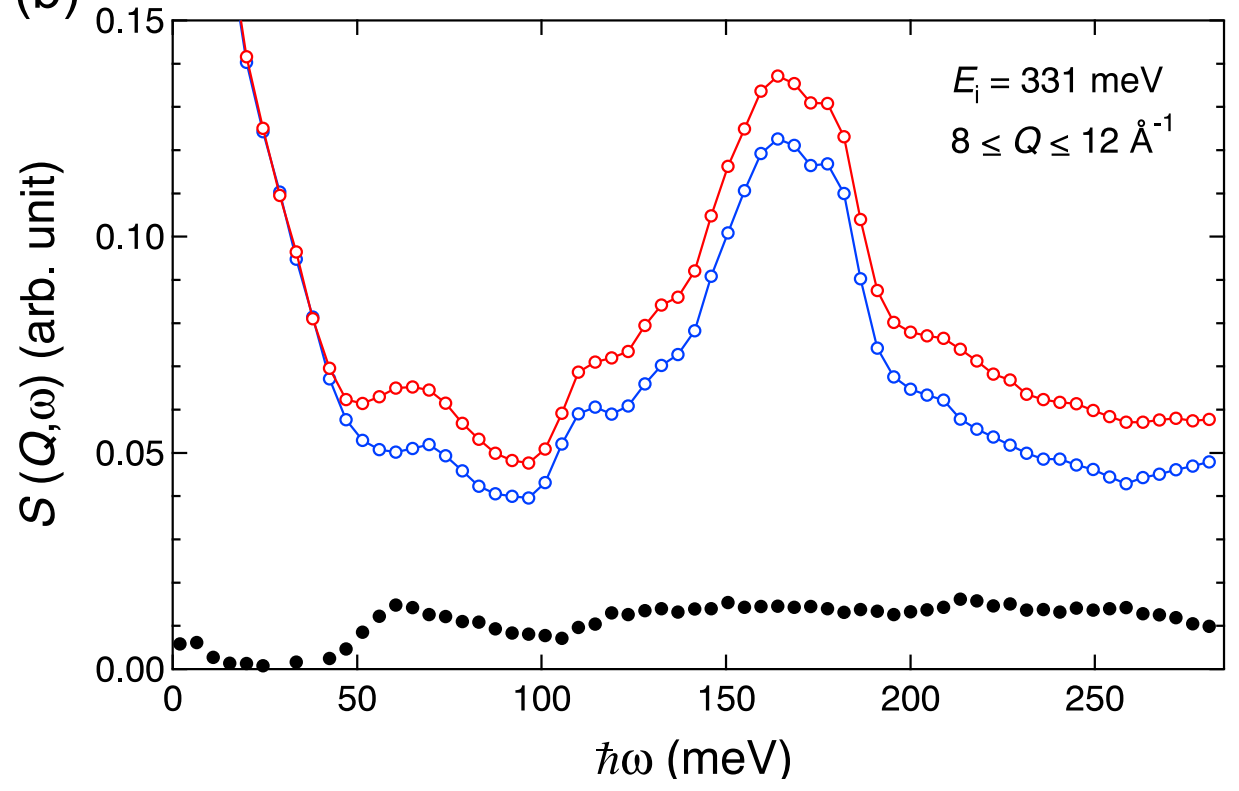

FIG. 3. (Color online) Inelastic neutron scattering spectra of nano- $\mathrm{PdH}_{0.42}$ and nano-Pd, recorded with $E_{\mathrm{i}} \mathrm{s}$ of (a) $168 \mathrm{meV}$ and (b) $331 \mathrm{meV}$ at $10 \mathrm{~K}$. The differences between the two spectra, $\left(\right.$ nano- $\left.\mathrm{PdH}_{0.42}\right)-($ nano-Pd), are also shown.

region. Additional excitations clearly appear above $80 \mathrm{meV}$ in nano- $\mathrm{PdH}_{0.42}$. It is noted that the position of the first peak slightly shifts toward a higher energy side in nano- $\mathrm{PdH}_{0.42}$, suggesting that the vibrational mode hardens. From the spectrum with $E_{\mathrm{i}}=168 \mathrm{meV}$, the peak position was estimated to be $59.3 \mathrm{meV}$ which is $3 \%$ higher than that observed in 

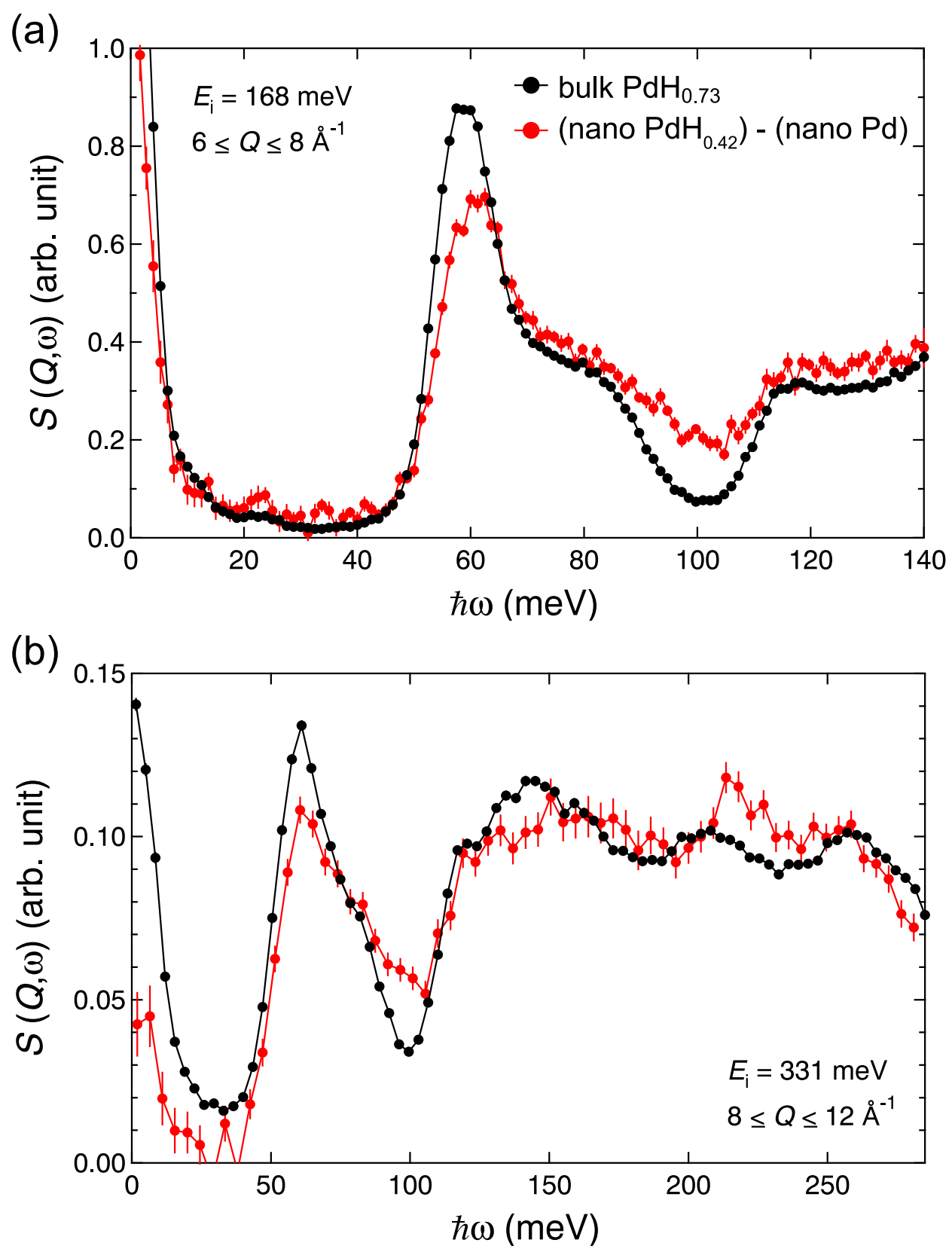

FIG. 4. (Color online) Inelastic neutron scattering spectra of bulk $\mathrm{PdH}_{0.73}$ and nano- $\mathrm{PdH}_{0.42}$ recorded with $E_{\mathrm{i}} \mathrm{s}$ of (a) $168 \mathrm{meV}$ and (b) $331 \mathrm{meV}$. The data of nano- $\mathrm{PdH}_{0.42}$, from which the scattering contribution from nano-Pd is subtracted, are multiplied by 7.3 for a comparison purpose.

bulk. The hardening could be related to the fact that the lattice constant of nano- $\mathrm{PdH}_{0.42}$ is smaller than that of bulk $\mathrm{PdH}_{0.73}$ [38]. It is also found that the vibrational excitations become broader in the nanocrystalline sample, implying an inhomogeneity of potential at $\mathrm{H}$ sites inside the nanoparticle. 


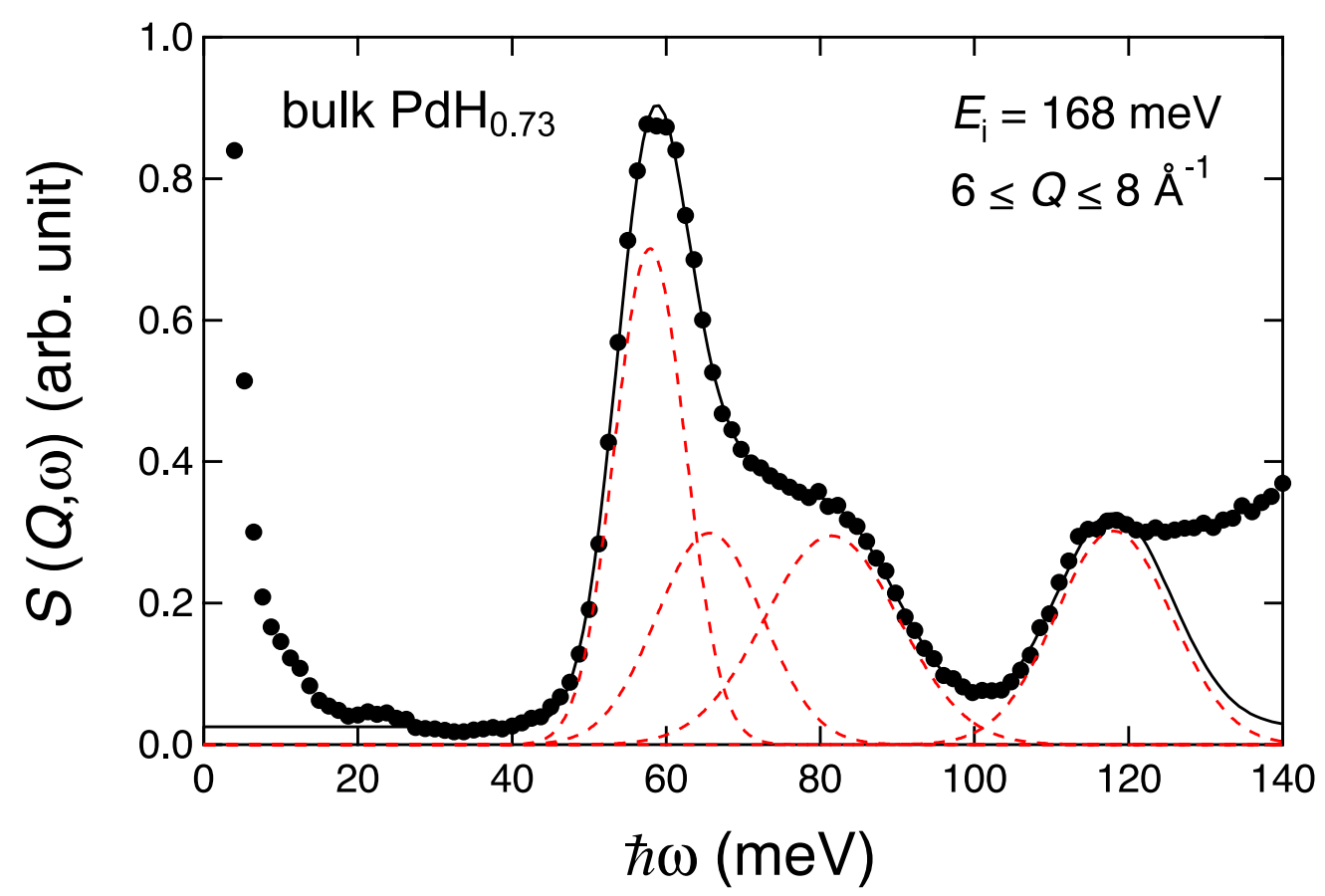

FIG. 5. (Color online) Inelastic neutron scattering spectrum of bulk $\mathrm{PdH}_{0.73}$ with $E_{\mathrm{i}}=168 \mathrm{meV}$ at $10 \mathrm{~K}$. Solid curves represents the result of the fit with multiple Gaussians. The dashed curve corresponds to each Gaussian function.

\section{B. Analysis for bulk $\mathrm{PdH}_{x}$}

In order to quantify the peak positions and areas, the INS spectra of bulk $\mathrm{PdH}_{0.73}$ were fitted with multiple Gaussians $G_{n, i}(\omega)$,

$$
\begin{aligned}
S(Q, \omega)_{\text {bulk }} & =\sum_{n} A_{n} \sum_{i} f_{n, i} G_{n, i}(\omega), \\
G_{n, i}(\omega) & =\sqrt{\frac{\ln 2}{\pi}} \frac{1}{\Gamma_{n, i}} e^{-\ln 2\left(\frac{\hbar \omega-E_{n, i}}{\Gamma_{n, i}}\right)^{2}},
\end{aligned}
$$

with a constraint of $\sum_{i} f_{n, i}=1$. Here the Gaussians were used to approximately describe the spectra. $E_{n, i}$ and $\Gamma_{n, i}$ are the position of the center and the half width at half maximum (HWHM) of each peak, respectively.

We first analyzed the data obtained with $E_{\mathrm{i}}=168 \mathrm{meV}$ to characterize the fundamental $(n=1)$ excitation mode. The data in an energy range between 34 and $122 \mathrm{meV}$ were fitted with four Gaussians; the fourth one is for $n=2$. The result of the fit is shown as a solid curve in Fig. 5. The positions of the three peaks for $n=1$ were 57.9, 65.6, and $81.5 \mathrm{meV}$. Next, advanced multi-peak fitting was carried out for the spectrum with $E_{\mathrm{i}}=331 \mathrm{meV}$ (See 
Fig. 6(a)). Nine Gaussian functions were used to reproduce the vibrational spectrum up to the $n=5$ oscillator mode. The individual peaks are displayed as dashed curves. The positions and widths of the first three peaks ( $n=1$ mode) were fixed to the values obtained from the fit for the data with $E_{\mathrm{i}}=168 \mathrm{meV}$.

In the quantum Harmonic oscillator (QHO) model [60], the scattering intensity and energy for the $n$th excitation are given by

$$
\begin{aligned}
& A_{n} \propto e^{-\left\langle u^{2}\right\rangle Q^{2}}\left(\left\langle u^{2}\right\rangle Q^{2}\right)^{n} / n ! \\
& E_{n}-E_{0}=n \hbar \omega_{0},
\end{aligned}
$$

where $\left\langle u^{2}\right\rangle\left(=\hbar / 2 m \omega_{0}\right)$ is the mean square displacement of zero-point vibration and $\omega_{0}$ the fundamental frequency. $n$ ! is replaced by the Gamma function $\Gamma(n+1)$ in a practical manner. Based on the QHO model, the peak position, width, and area of the $n=5$ mode were assumed as $\left(E_{5}-E_{0}\right)=5 / 4\left(E_{4}-E_{0}\right), \gamma_{5}=\gamma_{4}$, and $A_{5}=0.656 A_{4}$. These constraints were needed to analyze the excitation modes with $n \leq 4$ properly.

Figures 6(b) and (c) present $A_{n}$ and the "averaged" excitation energy against $n$. The averaged energies for $n=1,2$ were calculated as follows;

$$
\left\langle E_{n}\right\rangle-E_{0}=\sum_{i} f_{n, i} E_{n, i}
$$

where $f_{n, i}$ and $E_{n, i}$ are the same as those in Eq. (1).

The intensity and energy are sufficiently described by the QHO model, as shown by solid curves in Figs. 6(b) and 6(c). From the fit with Eq. (2), $\left\langle u^{2}\right\rangle$ and $\hbar \omega_{0}$ were estimated to be $0.0308(3) \AA^{2}$ and 68.7(9) meV, respectively. In the QHO model, the relation $\left\langle u^{2}\right\rangle=\hbar / 2 m \omega_{0}$ holds at low temperature. $\left\langle u^{2}\right\rangle$ is evaluated to be $0.0304 \AA^{2}$ for $\hbar \omega_{0}=68.7 \mathrm{meV}$ and is in excellent agreement with the above value from the fitting.

We now discuss the splitting of the excitation bands. The first band consists of a peak centered at $58 \mathrm{meV}$ and a shoulder at $81 \mathrm{meV}$ (see Fig. 5). In terms of $\mathrm{H}-\mathrm{H}$ interactions, the main peak is ascribed to a transverse optical mode, while the high-energy shoulder to a longitudinal one [49], The peak positions are consistent with the dispersion relation measured by INS $[42,46]$. However there is another interpretation; namely, the shoulder is a transition to the Franck-Condon level in the frozen Pd lattice, or without consideration of the lattice relaxation in the excited states $[50,52]$. The origin of the shoulder is still an open problem but is beyond the scope of this paper. 

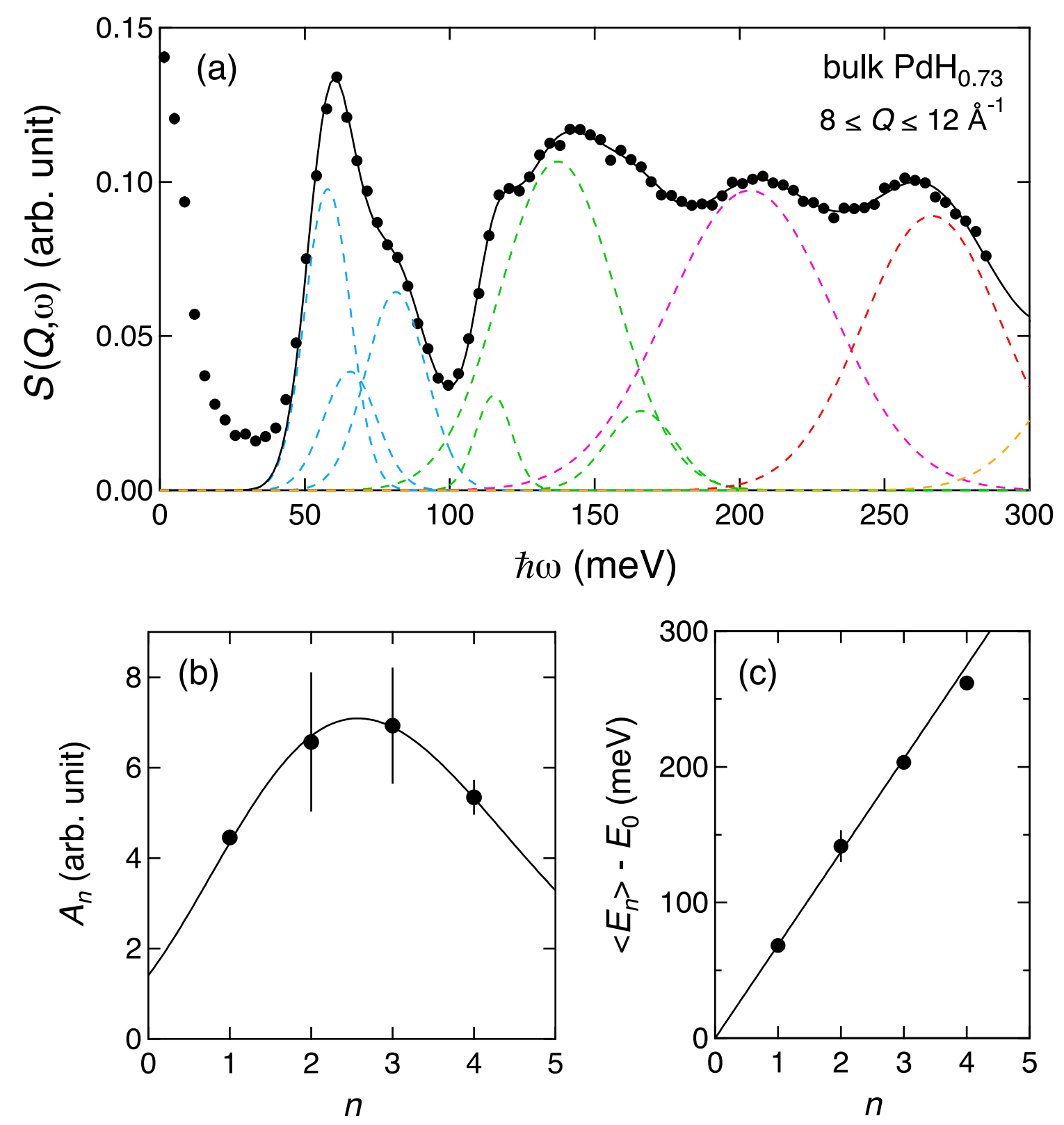

FIG. 6. (Color online) (a) Inelastic neutron scattering spectrum for bulk $\mathrm{PdH}_{0.73}$ at $10 \mathrm{~K}$. The cyan, green, magenta, red and orange dashed curves correspond to the $n=1,2,3,4,5$ excitation modes, respectively. (b) Intensity and (c) excitation energy against a quantum number $n$.

The split of the second band is considered to be due to anharmonic effects [45, 49-51]. For the three-dimensional isotropic harmonic oscillator, the Cartesian eigenstates $\left|n_{x} n_{y} n_{z}\right\rangle$ of the energy level $n\left(=n_{x}+n_{y}+n_{z}\right)$ are $\frac{1}{2}(n+1)(n+2)$-fold degenerate. The fourth-order anharmonic terms can lift the degeneracy of the states with $n \geq 2$. In fact, the $n=2$ state splits into three levels. The peak positions of the second band were estimated to be 
115, 137, and $167 \mathrm{meV}$, which are in reasonable agreement with the literature values [50]. As for the third and fourth excitation modes, the peak splitting was not clearly seen and the excitations were represented as single broad peaks. It should be stressed here that the "averaged" energy of the $n$ state is the same as that for QHO within the framework of the perturbation theory. Additionally, the anisotropy in the INS spectra [49, 50], which manifests itself by the anharmonicity, is not prominent in powder samples.

\section{Analysis for nano-PdH ${ }_{x}$}

Figure $7(\mathrm{a})$ shows the INS spectrum of nano- $\mathrm{PdH}_{0.42}$. The spectrum was fitted with the combination of the spectrum of the bulk $\mathrm{PdH}_{0.73}$ (dashed curve) and the additional excitations (solid curves),

$$
\begin{aligned}
& S(Q, \omega)_{\text {nano }}=b_{0} S(Q, \omega)_{\text {bulk }}+b_{1} S(Q, \omega)_{\text {add }}, \\
& S(Q, \omega)_{\text {add }}=\sum_{n^{\prime}} A_{n^{\prime}} G_{n^{\prime}}(\omega) .
\end{aligned}
$$

The additional component was represented by multiple Gaussians $G_{n^{\prime}}(\omega)$. In the fitting analysis, the parameters for $S(Q, \omega)_{\text {bulk }}$ were fixed to be the same as those obtained for bulk $\mathrm{PdH}_{0.73}$, except the $1_{1}$ mode. As stated in Sec. III.A, the $1_{1}$ mode slightly hardens in nano- $\mathrm{PdH}_{0.42}$. The parameters for the mode were estimated from the fit of the spectrum taken with $E_{\mathrm{i}}=168 \mathrm{meV}$. Thus, the parameters to be determined in the fitting were the prefactors $b_{0}, b_{1}$, areas, peak positions, and widths of $S(Q, \omega)$ add the widths for the $n=2$, 3, 4 excitations are constrained to be the same. The fraction of the additional component, $b_{1} /\left(b_{0}+b_{1}\right)$, was estimated to be 0.22 . The value is consistent with the fraction of $\mathrm{D}$ atoms occupying the $T$ site $(\approx 0.25$ at $44 \mathrm{~K})$ estimated from the neutron diffraction study [38]. It is naturally concluded that that the bulk-like excitations are the vibrations of $\mathrm{H}$ atoms at the $O$ sites mainly in the interior region while the additional one originates from the $\mathrm{H}$ atoms at the $T$ sites in the subsurface. The stability of the $T$ site should depend on the mass (H or D) due to the zero point energy. However, we consider that this isotope effect is not so serious to demolish this conclusion. Of course it is necessary to conduct the NPD experiment for $\mathrm{PdH}$ though it is very difficult because of small coherent and larger incoherent scattering cross sections of a $\mathrm{H}$ atom.

The estimated intensities and averaged excitation energies are displayed in Fig. 7(b) and 

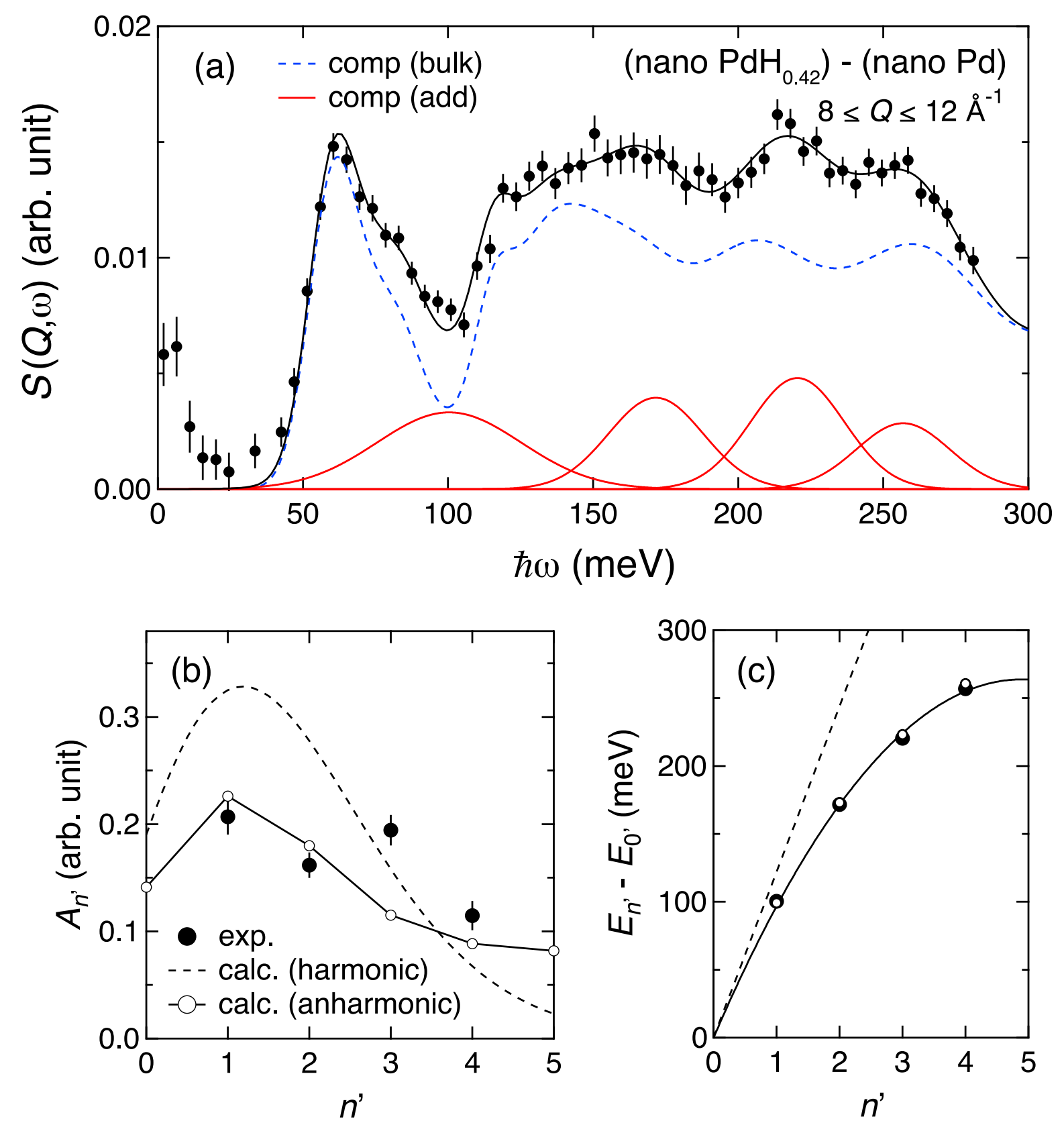

FIG. 7. (Color online) (a) Inelastic neutron scattering spectrum for nano- $\mathrm{PdH}_{0.42}$ at $10 \mathrm{~K}$. The scattering contribution from nano-Pd is subtracted. (b) Intensity and (c) excitation energy against a quantum number $n$.

(c). Dashed curves represent the expectation from the QHO model with $\hbar \omega_{0}=122 \mathrm{meV}$. Obviously, the QHO model does not reproduce the experimental results. In order to characterize the additional states, anharmonicity should be considered.

Finally, we discuss the anharmonic potential by a simple numerical method. We assumed 


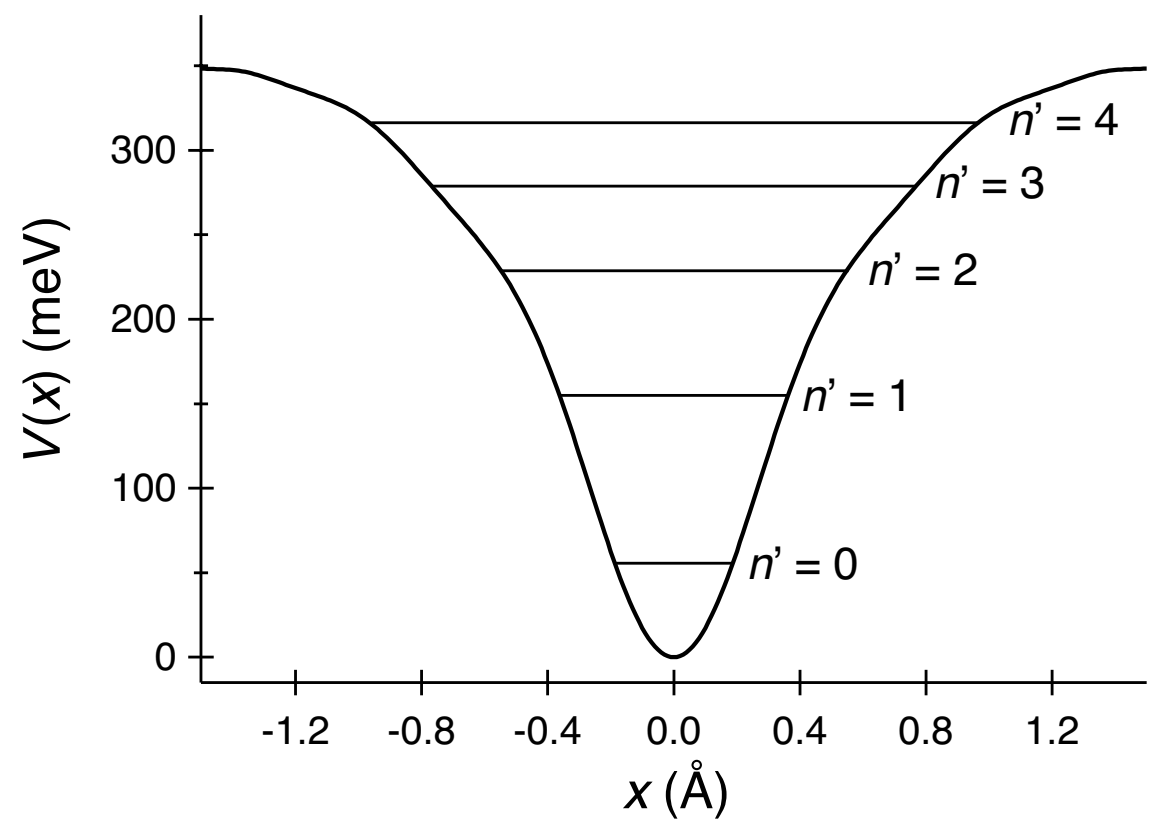

FIG. 8. Potential energy as a function of the atomic displacements relative to the equilibrium position of the $\mathrm{H}$ atom. The curve is calculated using Eq. (5). The parameters $\omega_{0}$ and $c_{2 i}$ used in the calculation are summarized in Table. I. Horizontal lines represent the eigenvalues $E_{n^{\prime}}$.

a one-dimensional symmetric polynomial function,

$$
V(x)=\frac{1}{2} m \omega_{0} x^{2}+\sum_{i=2}^{7} c_{2 i} x^{2 i} .
$$

The first term is just the QHO potential for which an exact analytical solution is known. Therefore, the 20 lowest wavefunctions for the QHO were chosen as a basis set in our calculation. By diagonalizing the Hamiltonian, $\mathcal{H}=-\frac{\hbar^{2}}{2 m} \frac{d^{2}}{d x^{2}}+V(x)$, eigenvalues $E_{n^{\prime}}$ and eigenstates $\psi_{n^{\prime}}$ for the anharmonic potential were obtained. The scattering intensities were calculated with the following formula;

$$
A_{n^{\prime}} \propto\left|\int_{-\infty}^{\infty} \psi_{n^{\prime}} e^{i Q x} \psi_{0} d x\right|^{2} .
$$

These calculations were carried out using scripts written in Mathematica 10.4 software (Wolfram Research).

Open circles in Fig. 7(b) and (c) are the calculated values with assuming the potential surface shown in Fig. 8. The reasonable agreement is obtained between the experiment and calculation. The large deviation at $n^{\prime}=3$ could be due to the presence of vibration of $\mathrm{H}$ atoms on the $\mathrm{Pd}$ surface. The deduced potential surface is highly anharmonic and 
TABLE I. Parameters for the anharmonic potential, $V(x)=\frac{1}{2} m \omega_{0} x^{2}+\sum_{i=2}^{7} c_{2 i} x^{2 i}$. All values are in $\mathrm{meV}$.

\begin{tabular}{ll}
\hline \hline$\hbar \omega_{0}=122 \mathrm{meV}$ & $c_{12}=-4864.1 \mathrm{meV} / \AA^{2}$ \\
$c_{4}=-5970 \mathrm{meV} / \AA^{2}$ & $c_{14}=1257 \mathrm{meV} / \AA^{2}$ \\
$c_{6}=12054 \mathrm{meV} / \AA^{2}$ & $c_{16}=-151.01 \mathrm{meV} / \AA^{2}$ \\
$c_{8}=-14593 \mathrm{meV} / \AA^{2}$ & $c_{18}=-0.38007 \mathrm{meV} / \AA^{2}$ \\
$c_{10}=10802 \mathrm{meV} / \AA^{2}$ & $c_{20}=1.2804 \mathrm{meV} / \AA^{2}$ \\
\hline \hline
\end{tabular}

of trumpet-like shape, in contrast to the nearly harmonic potential in bulk $\mathrm{PdH}_{0.73}$. The potential is steep at lower energies while rather gentle at higher energies. Compared with the harmonic model, $A_{n^{\prime}}$, which reflects the spatial distribution of the wavefunctions, represents a larger value for larger $n$.

The fundamental peak energy $\hbar \omega_{0}$ was estimated to be $122 \mathrm{meV}$ which is significantly larger than that of bulk $\mathrm{PdH}_{0.73}$. The vibrational mode comes from the $\mathrm{H}$ atom in a steep potential. Such a remarkable change in $\omega_{0}$ cannot be explained by the change in the lattice constant. The present analysis suggests that the distance between $\mathrm{H}$ and $\mathrm{Pd}$ atoms is rather short. In fact, the $\mathrm{H}-\mathrm{Pd}$ distance is $2 \AA$ for the $O$ site and $1.73 \AA$ for the $T$ site. The observation of the vibrational modes with high fundamental energy also support our conclusion that some of $\mathrm{H}$ atoms are accommodated at the $T$ sites in the nanoparticles. It has been pointed out that the vibrational energy (or zero point energy) at the $T$ site is higher than that at the $O$ site $[51,61,62]$. However, the anharmonicity is not predicted for the $T$ site in bulk $\mathrm{PdH}_{x}$. We argue that the newly appeared vibrational states with strong anharmonicity in nano- $\mathrm{PdH}_{0.42}$ are characteristic of the $\mathrm{H}$ atoms at the $T$ site in the subsurface. The strong modification of potential could be caused by surface strain/distortion effects of nanoparticles.

\section{SUMMARY}

Vibrational spectra of hydrogen in bulk and nanocrystalline $\mathrm{Pd}$ were investigated by inelastic neutron scattering in the energy range up to $300 \mathrm{meV}$. The spectrum of bulk $\mathrm{PdH}_{0.73}$ is roughly described by quantum harmonic oscillators. In $\mathrm{PdH}_{0.42}$ nanocrystals 
with a diameter of $8 \mathrm{~nm}$, the excess excitations were found at higher energies in addition to the excitations which closely resemble those observed in bulk $\mathrm{PdH}_{0.73}$. Our analysis demonstrated that the excess excitations are attributed to the hydrogen vibrations in a highly anharmonic trumpet-like potential which is realized at the $T$ sites in the subsurface region. Further theoretical calculations on the actual three-dimensional nanocrystal are needed to reveal the details of vibrational states.

\section{ACKNOWLEDGMENTS}

This work was supported by Core Research for Evolutional Science and Technology (CREST) from Japan Science and Technology Agency (JST). We thank Prof. S. Ikeda

for fruitful discussions, and Dr. R. Kajimoto and Dr. Y. Inamura for their help in the experiment on 4 SEASONS. The experiment in the MLF at J-PARC was performed with the approval of J-PARC (Proposal No. 2015A0260).

[1] G. Schmid, Clusters and Colloids (VCH, Weinheim,1994).

[2] Y. Volokitin, J. Sinzig, L. J. de Jongh, G. Schmid, M. N. Vargaftik and I. I. Moiseevi, Nature 384, 621 (1996).

[3] A. Pundt and R. Kirchheim, Annu. Rev. Mater. Res. 36, 555 (2006).

[4] J. M. Campelo, D. Luna, R. Luque, J. M. Marinas, and A. A. Romero, ChemSusChem 2, 18 (2009).

[5] R. J. Wolf, M. W. Lee, and J. R. Ray, Phys. Rev. Lett. 73, 557 (1994).

[6] C. Sachs, A. Pundt, R. Kirchheim, M. Winter, M. T. Reetz, and D. Fritsch, Phys. Rev. B 64, 075408 (2001).

[7] V. Bérubé, G. Radtke, M. Dresselhaus, and G. Chen, Int. J. Energy Res. 31, 637 (2007).

[8] M. Yamauchi, R. Ikeda, H. Kitagawa, and M. Takata, J. Phys. Chem. C 112, 3294 (2008).

[9] R. Bardhan, L. O. Hedges, C. L. Pint, A. Javey, S. Whitelam, and J. J. Urban, Nature Mater. 12, 905 (2013).

[10] G. Li, H. Kobayashi, J. M. Taylor, R. Ikeda, Y. Kubota, K. Kato, M. Takata, T. Yamamoto, S. Toh, S. Matsumura, and H. Kitagawa, Nature Mater. 13, 802 (2014). 
[11] G. Li, H. Kobayashi, S. Dekura, R. Ikeda, Y. Kubota, K. Kato, M. Takata, T. Yamamoto, S. Matsumura, and H. Kitagawa, J. Am. Chem. Soc. 136, 10222 (2014).

[12] R. Griessen, N. Strohfeldt, and H. Giessen, Nature Mater. 15, 311 (2016).

[13] D. Teschner, J. Borsodi, A. Wootsch, Z. Révay, M. Hävecker, A. Knop-Gericke, S. D. Jackson, and D. Schlögl, Science 320, 86 (2008).

[14] A. Mohanty, N. Garg, and R. Jin, Angew. Chem. Int. Ed. 49, 4962 (2010).

[15] S. Ogura, M. Okada, and K. Fukutani, J. Phys. Chem. 119, 23973 (2015).

[16] J. Mondal, Q. T. Trinh, A. Jana, W. K. H. Ng, P. Borah, H. Hirao, and Y. Zhao, ACS Appl. Mater. Interfaces 8, 15307 (2016).

[17] K. H. Rieder, M. Baumberger, and W. Stocker, Phys. Rev. Lett. 51, 1799 (1983).

[18] R. J. Behm, V. Penka, M.-G. Cattania, K. Christmann and G. Ertl, J. Chem. Phys. 78, 7486 (1983).

[19] H. Okuyama, W. Siga, N. Takagi, M. Nishijima, and T. Aruga, Surf. Sci. 401, 344 (1998).

[20] M. Wilde and K. Fukutani, Phys. Rev. B 78, 115411 (2008).

[21] M. Wilde, K. Fukutani, M. Naschitzki, and H. J. Freund, Phys. Rev. B 77, 113412 (2008).

[22] S. Wilke, D. Hennig, and R. Löber, Phys. Rev. B 50, 2548 (1994).

[23] J.-F. Paul and P. Sautet, Phys. Rev. B 53, 8015 (1996).

[24] N. Ozawa, N. B. Arboleda Jr., T. A. Roman, H. Nakanishi, W. A. Diño, and H. Kasai, J. Phys. Condens. Matter 19, 365214 (2007).

[25] H. Frieske and E. Wicke, Ber. Bunsenges. Phys. Chem. 77, 48 (1973).

[26] F. D. Manchester, A. San-Martin, and J. M. Pitre, J. Phase Equilibria 15, 62 (1994).

[27] J. E. Worsham, M. K. Wilkinson, and C. G. Shull, J. Phys. Chem. Solids 3, 303 (1957).

[28] G. A. Ferguson, A. J. Schindler, T. Tanaka, and T. Morita, Phys. Rev. 137, A483 (1965).

[29] G. Nelin, Phys. Stat. Sol. 45, 527 (1971).

[30] I. S. Anderson, C. J. Carlile, and D. K. Ross, J. Phys. C 11, L381 (1978).

[31] O. Blaschko, R. Klemencic, P. Weinzierl, and O. Eder, Solid State Commun. 27, 1149 (1978).

[32] T. E. Ellis, C. B. Satterthwaite, M. H. Mueller, and T. O. Brun, Phys. Rev. Lett. 42, 456 (1979).

[33] O. Blaschko, R. Klemencic, P. Weinzierl, and O. Eder, Acta Cryst. A36, 605 (1980).

[34] S. J. Kennedy, E.Wu, E. H. Kisi, E. M. Gray, and B. J. Kennedy, J. Phys.: Condens. Matter 7, L33 (1995). 
[35] E. Wu, S. J. Kennedy, E. M. Gray, and E. H. Kisi, J. Phys.: Condens. Matter 8, 2807 (1996).

[36] M. Pitt and E. M. Gray, Europhys. Lett. 64, 344 (2003).

[37] K. G. McLennan, E. M. Gray, and J. F. Dobson, Phys. Rev. B 78, 014104 (2008).

[38] H. Akiba, M. Kofu, H. Kobayashi, H. Kitagawa, K. Ikeda, T. Otomo, and O. Yamamuro, J. Am. Chem. Soc. 138, 10238 (2016).

[39] M. Kofu, N. Hashimoto, H. Akiba, H. Kobayashi, H. Kitagawa, M. Tyagi, A. Faraone, J. R. D. Copley, W. Lohstroh, and O. Yamamuro, Phys. Rev. B 94, 064303 (2016).

[40] J. Bergsma and J. A. Goedkoop, Physica (Amsterdam) 26, 744 (1960)

[41] M. R. Chowdhury and D. K. Ross, Solid State Commun. 13, 229 (1973).

[42] J. M. Rowe, J. J. Rush, H. G. Smith, M. Mostoller, and H. E. Flotow, Phys. Rev. Lett. 33, 1297 (1974).

[43] A. Rahman, K. Sköld, C. Pelizzari, S. K. Sinha, and H. Flotow, Phys. Rev. B 14, 3630 (1976).

[44] W. Drexel, A. Murani, D. Tocchetti, W. Kley, I. Sosnowska, and D. K. Ross, J. Phys. Chem. Solids 37, 1135 (1976).

[45] J. J. Rush, J. M. Rowe, and D. Richter, Z. Phys. B 55, 283 (1984).

[46] J. M. Rowe, J. J. Rush, J. E. Schirber, and J. M. Mintz, Phys. Rev. Lett. 57, 2955 (1986).

[47] A. I. Kolesnikov, I. Natkaniec, V. E. Antonov, I. T. Belash , V. K. Fedotov, J. Krawczyk, J. Mayer, and E. G. Ponyatovsky, Physca B 174, 257 (1991).

[48] Y. Nakai, E. Akiba, H. Asano, and S. Ikeda, J. Phys. Soc. Jpn. 61, 1834 (1992).

[49] D. K. Ross, V. E. Antonov, E. L. Bokhenkov, A. I. Kolesnikov, E. G. Ponyatovsky, and J. Tomkinson, Phys. Rev. B 58, 2591 (1998).

[50] M. Kemali, J. E. Totolici, D. K. Ross, and I. Morrison, Phys. Rev. Lett. 84, 1531 (2000).

[51] C. Elsässer, K. M. Ho, C. T. Chan, and M. Fähnle, J. Phys.:Condens. Matter 4, 5207 (1992).

[52] H. Krimmel, L. Schimmele, C. Elsässer, and M. Fähnle, J. Phys.: Condens. Matter 6, 7679 (1994).

[53] U. Stuhr, H. Wipf, T. J. Udovic, J. Weißmüller, and H. Gleiter, J. Phys.: Condens. Matter 7, 219 (1995).

[54] H. Akiba, H. Kobayashi, H. Kitagawa, M. Kofu, and O. Yamamuro, Phys. Rev. B 92, 064202 (2015).

[55] B. Lim, M. Jiang, P. H. C. Camargo, E. C. Cho, J. Tao, X. Lu, Y. Zhu, and Y. Xia, Science 324, $1302(2009)$. 
[56] R. Kajimoto et al., J. Phys. Soc. Jpn. 80, SB025 (2011).

[57] M. Nakamura, R. Kajimoto, Y. Inamura, F. Mizuno, M. Fujita, T. Yokoo, and M. Arai J. Phys. Soc. Jpn. 78, 093002 (2009).

[58] Y. Inamura, T. Nakatani, J. Suzuki, and T. Otomo, J. Phys. Soc. Jpn. 82 SA031 (2013).

[59] Y. Borodko, S. E. Habas, M. Koebel, P. Yang, H. Frei, and G. A. Somorjai, J. Phys. Chem. B 110, $23052(2006)$.

[60] S. W. Lovesey, Theory of Neutron Scattering from Condensed Matter, Vol. 1 (Clarendon, Oxford, 1984).

[61] R. Caputo and A. Alavi, Mol. Phys. 101, 1781 (2003).

[62] C. Wei, F. T. Kong, and H. R. Gong, Int. J. Hydrog. Energy 38, 16485 (2013). 